\title{
Discussion on Access Database Network Teaching Mode
}

\section{Xin Sheng}

Qilu University of Technology, Shandong Jinan 250353, China.

\begin{abstract}
Access is a database application development tool software, which is mainly used in the management of most small and medium-sized enterprises and has very high practical application value. When teaching Access database, teachers should combine multiple teaching methods to improve the teaching effect of Access database. Aiming at the various problems in Access database teaching, this paper conducts research and discussion on the reform of Access database network teaching mode, hoping to bring some new inspiration to Access software teaching.
\end{abstract}

Keywords: Access database; network teaching; model discussion

With the popularization of computer technology, more and more small and medium-sized enterprises apply computer technology in business management, among which Access database is one of the most practical software. When teaching Access database, because its application theory is relatively abstract, it is difficult for students to master and use it flexibly in limited classroom time. Therefore, in order to improve the teaching efficiency of Access database, teachers should adopt more effective and innovative teaching methods. Combining my own teaching experience and starting from the actual teaching situation of Access database, the author analyzes and discusses access database network teaching methods, and proposes some effective teaching reform measures, hoping to improve students' enthusiasm for learning Access database, and make students truly proficient Master the application of Access software to lay a solid foundation for further study and work in the future.

\section{The function and role of Access database}

Access database is a relational database management system issued by Microsoft Corporation. Like other Microsoft Office software, Access database is an object-oriented programming language that can link various objects and corresponding program components to each other. The required content is presented visually, which is very convenient and practical. The specific purpose of the Access database is reflected in the following two aspects:

First, it is used for various data analysis. Access has very powerful data processing and data statistics capabilities. The query function of Access software can quickly and effectively retrieve various data. This is particularly prominent in a large amount of data analysis, which greatly improves work efficiency and effectiveness.

Second, it is used to remember simple software development within the enterprise. In enterprise management, production management, sales management, inventory management and various management software are inseparable from the support of Access. Its biggest advantage is that it is easy to learn, and the learning cost and efficiency are very high. It is very helpful to enterprise management staff. A very important helper in enterprise development.

\section{Problems in Access database teaching}

\subsection{Access database teaching content is not compact enough}

Access database is less difficult to learn than other database software, but it still needs to learn a lot of basic theoretical knowledge and follow-up application development. It still requires considerable effort to use Access 
database proficiently. In actual teaching, teachers often disperse each knowledge point to explain one by one, and do not pay attention to the relevance of knowledge points. In addition, the content of knowledge is highly abstract, which makes it difficult for students to form a complete knowledge structure in their minds. It is difficult for students to find out the problem in the first time when dealing with practical problems, which greatly weakens the learning efficiency of Access database.

\subsection{Lack of pertinence in the learning process}

Access database needle is a software that solves practical problems in business management. Different learners have different actual needs for Access database. In actual teaching, teachers did not give targeted explanations according to the different needs of students, and taught Most of the time is spent on the study of basic theoretical knowledge, and the content of specific practical exercises is less, making it difficult for students to learn useful knowledge for themselves, which greatly reduces the enthusiasm of students in learning.

\section{Research on Access database network teaching mode}

Traditional Access database teaching has problems such as unclear educational purpose, limited by practice and space teaching, limited communication between students and teachers, and lack of specific practical training projects. These problems are taught through the Internet It can be changed to a large extent, greatly improving the enthusiasm of students in learning, and improving the teaching efficiency of Access database.

First of all, online teaching can give students great freedom. The content of traditional Access database teaching methods is not compact enough, and students cannot quickly form a complete knowledge structure system. With network teaching, teachers can classify and integrate all knowledge content, and students can see a complete knowledge system before learning. Special repetitive learning for their weak local memory improves the effectiveness of Access database teaching.

Secondly, online teaching eliminates the limitations of time and space. Access database learning is very compact. If you miss a class accidentally, it will have a great impact. And because of the function of recording and reviewing online lectures, and there is no special geographical restriction, students can study anytime and anywhere as long as they have the Internet. And watching, improve the access database learning and dissemination way, and allow students to review the past and learn the new, improve the efficiency of students' learning.

In addition, online teaching content is very extensible. Teachers can record multiple practical teaching projects in advance to show the practical content that students care about one by one, taking into account the diverse needs of students to the greatest extent, and allowing students to carry out according to their own needs. The choice reflects the advantages of online teaching focusing on individualized development, greatly improving students' enthusiasm for independent learning, and making Access database teaching more efficient.

Finally, the implementation of Access database network teaching can make the teaching test more flexible. Online teaching is more open and flexible for the testing and practice of teaching content. After the teacher arranges the homework and test content, students can answer the questions through the online teaching platform, and quickly get the feedback of the answers, check the deficiencies in time, and can According to the convenience of network information exchange, communicate with teachers and other students at any time, solve problems as quickly as possible, and improve their understanding of Access database.

\section{Concluding remarks}

In summary, Access database has a very high application rate in contemporary enterprise management, and it is one of the indispensable application software in enterprise management. In order to improve the teaching efficiency of Access database and ensure its teaching quality, teachers should combine the actual situation of students Circumstances, on the basis of traditional teaching methods, use the advantages of online teaching to stimulate students' learning enthusiasm, close the relationship between teachers and students, actively communicate with students, follow up students' learning in a timely manner, and use online teaching Data feedback is used to adjust the memory of teaching 
content to improve students' practical application ability.

\section{References}

1. Li Rong, Feng Suqin, Li Zhaoxia. Research and practice of ACCESS teaching mode reform for non-computer majors*[J]. Computer Times, 2013(6): 62-63.

2. Yu Ning, Cui Wuzi. Analysis and Discussion on the Network Course Design of "Access Database Programming"[J]. Journal of Beijing Union University (Natural Science Edition), 2010. (09)-63.

3. Ning Haitao. Exploration and Research on the Teaching Reform of Access Database [J]. Computer Knowledge and Technology, 2015, 11(020):116-117. 